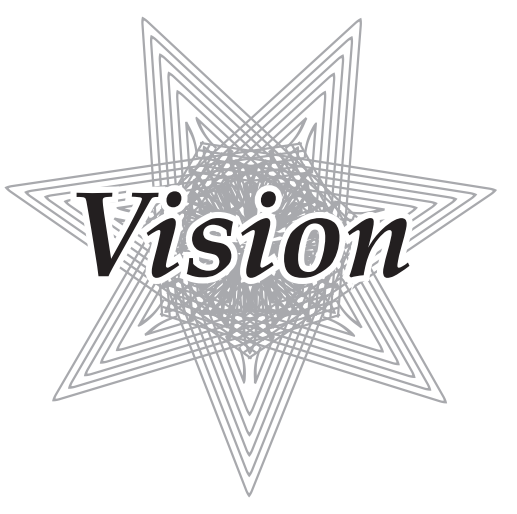

\title{
Resurgence of Solid-state Ionic Conductor Research is Growing the Field of Electrochemistry
}

\section{Jeff SAKAMOTO}

Over 50 years ago, physicists, ceramists and solid-state electrochemists were developing alkali metal fast ion conductors (sodium beta alumina) to enable rechargeable batteries for electric vehicles. Although these batteries did not achieve widespread adoption, the research spawned the modern era of solid-state electrochemistry emphasizing alkali metal conducting materials research. The rapid evolution of this field in the 1970s and 1980s inspired the development of mixed conducting cathodes (e.g. $\mathrm{LiCoO}_{2}$ and related layered compounds) for Li-ion technology.

In the 1980s to $1990 \mathrm{~s}$, researchers were developing thin film batteries using LIPON. These batteries could achieve $>20,000$ cycles, demonstrating a ceramic electrolyte could physically and chemically stabilize the metallic Li anode interface and, more importantly, that Li solid-state batteries work.

In the last two decades, the discovery of several new bulk-processed fast ion conducting Li-ion solid electrolytes invigorated the field of solid-state electrochemistry. The new generation of solid-state electrolytes includes a broad range of chemistries such as sulfides, oxides, borates, polymers and polymer composites. Currently, room temperature Li-ion conductivities are approaching that of liquid electrolytes (e.g. sulfides and polymers) and a subset of solid-state electrolytes are also stable against Li metal and stable in ambient air. This new class of solid electrolytes comprises another new generation of solid-state electrochemistry research a half a century after the pioneering work on sodium beta alumina and several decades after the development of thin film Li metal batteries. However, compared to previous times, the battery landscape is dramatically different today....

The demand for low cost $(<\$ 100 / \mathrm{kWh})$ safe (non-flammable), high performance $(>1000 \mathrm{Wh} / \mathrm{l})$ is unprecedented. Governments and the auto industry are considering and preparing for a dramatic shift to supplant internal combustion engine power trains with battery-electric power trains. Similarly, miniaturization and longer operation time between charges is pushing the limits of batteries in microelectronics. State-of-the-art Li-ion technology works extremely well and currently is the leading battery technology. However, whether or not Li-ion can meet energy density, safety and cost requirement remains to be determined. Given these circumstances, and the significant role that batteries will play in shaping major technologies in the $21 \mathrm{st}$ century, urgency should add additional impetus to accelerate technological advancement unlike what was witnessed in past solidstate electrochemistry eras.

Combining the knowledge and experience gained over the decades with the new generation of materials, positions the broader electrochemistry, physics, materials science, mechanical engineering and manufacturing communities to push forward with a multi-faceted approach to accelerate the development of solid-state battery technology. Though there are several fast-ion conductors, the continued development of single and divalent fast-ion conductors with increased electrochemical and chemical stability and are manufacturable and mechanically robust is still needed. While there has been tremendous progress in achieving metal-electrolyte (solid-solid) interface resistance comparable to state-of-the-art Li-ion, cathode-electrolyte (solid-solid) interfaces still required dramatic improvements in kinetics and electrochemical and mechanical stability. As prototype cells made in laboratories demonstrate performance metrics, large-scale manufacturing becomes more and more important; yet, commercialization and manufacturing feasibility of solid-state batteries is currently an unknown. Similarly, manufacturing of thin film solid electrolytes at low cost and low energy intensity must be developed. In addition, the experience and expertise from the fuel cell community will likely benefit efforts to develop solid-state batteries, especially with regard to ceramic processing and cell design and integration.

Looking forward, electrochemical technologies could play a pivotal role in transitioning from fossil fuels to renewable electrical energy. Advanced batteries such as solid-state batteries are one example of a technology that is pushing the limits of conversion from chemical to electrical energy. However, fundamental research, creativity and the sharing of ideas will be necessary to overcome the technological challenges that lie ahead.

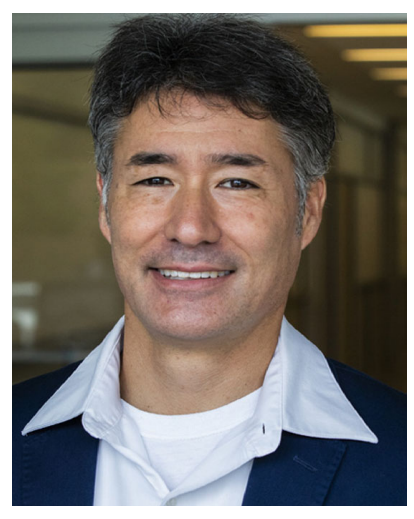

Jeff SAKAMOTO

Professor of Mechanical

Engineering, Materials

Science and Engineering, and

Macromolecular Science and Engineering

University of Michigan

Ann Arbor, Michigan, U.S.A. 